I have tried to see whether it might not be due to the falling of the object on the blind spot, but uniformly discover that it is not, as the disappearing object may be on the side of the retina opposite the blind spot. On careful experiment and observation I find that the disappearance is directly related to the degree of abstraction, and that I can reproduce it artificially, if I am successful, as I sometimes am, in effecting the abstraction necessary and at the same time the proper adjustment of attention. It is difficult to produce the artificial abstraction required, but when I am successful I effect the disappearance of the object, which immediately reappears the moment attention is given to it without altering the fixation of the eyes. The effect seems to be that of making clear an actual impression, while attention in the previous experiment seems to destroy an after image."

Hyslop's use of the term 'abstraction' in contrast with 'attention' is interesting, and supports the view of several correspondents, particularly Dr. N. Moray of Sheffield, that the word 'attention' in my communication in Nature is a source of difficulty. It was of course with this possibility in mind that I had written "attend to one of the objects and try to see or describe it in detail".

Prof. O. L. Zangwill has suggested that the mechanism of disappearance is related to Hering's local adaptation, but while this may be so, I find no evidence of Hering having associated local adaptation with attention.

Institute of Experimental Psychology,

University of Oxford,

1 South Parks Road, Oxford.

${ }^{1}$ Aubert, H., Moleschotts Untersuchungen zur Naturl., B2V, 279 (1858). ${ }^{2}$ Baird, J. W. The Color Sensitivity of the Peripheral Retina (Wash., Carnegie Inst., 1905).

${ }^{3}$ Helmholtz, H. von, Physiological Optics, 1924 ed., 2, 42 and elsewhere $(1866)$.

-Troxler, D., Himly and Schmidt, Opthalmologische Bibliothek, 2, 2 (1804).

'Aubert, H., and Förster, O., Allg. med. Zentralzeitung, Berlin, No. 33,259 (as summarized in a sentence by Helmholtz) (1957).

- Hyslop, J. H., Psychol. Rev., 10, 296 (1903).

' Hering, E., Grundzuge der Lehrer von Lichtsinn, 265 (1920), and Archiv für Ophthalm. (Graefe), 37, Abt. III, 31 (1891).

\section{Centrally Produced Geometrical Illusions}

So little is known about the mechanism responsible for the well-known geometrical illusions that it is not possible to decide on general theoretical grounds whether they are the result of retinal processes, central processes or both. An experimental procedure to demonstrate that they are at least partly of central origin is therefore of interest.

The procedure consists of constructing a visual target, in which the illusion figure is seen in stereoscopic vision but not in monocular vision. The simplest way of achieving this is as follows. A sheet of glass is covered with an array of randomly placed. dots. On a second glass sheet the well-known MüllerLyer illusion, for example, is constructed out of dotted lines, the separation of the dots being about the same as those on the first sheet. The two are then mounted parallel to one another, separated by a few inehes. When viewed monocularly under conditions which prevent head movements, shadows and other sources of monocular distance clues, the two planes are seen as merged and the Müller-Lyer figure cannot be distinguished. In binocular vision the two planes are easily separated and all subjects report the illusion.

The rigour of the experiment can be improved in two ways: (1) if the three vertices $A, B, C$, of the figure are marked by dots of a different colour, judgment of distance $A B$ compared with distance $B C$ can be made, and in fact turns out to be significantly different, under monocular and binocular conditions; (2) instead of using two real planes, the situation can be simulated by constructing stereoscope diagrams using an electronic computer ${ }^{1}$. This enables a rigorous control of factors such as dot density, and ensures that the signal received by each eye is a thoroughly pseudo-random array of dots; the information used by the stereoseopic mechanism is entirely contained in the relation between the two arrays.

A quantitative comparison of illusions produced in this way with their more familiar form involves intricate statistics and will be reported elsewhere. Figural after-effects can be produced under similar conditions, but are always weak, though I believe that this is due to incidental aspects of the procedure.

The work described here is part of the research programme of the National Physical Laboratory and this communication is published by permission of the Director of the Laboratory.

Autonomics Division,

S. PAPERT

National Physical Laboratory, Teddington, Middlesex.

1 Julesz, B., Proc. Fourth London Symp. on Information Theory, edit. C. Cherry, 1961.

\section{MISCELLANY}

\section{The National Reference Library of Science and Invention}

THE leading article in Nature of July 1 presents a timely challenge to all organizations concerned with the use of scientific and technical knowledge to think deeply about the plans for the National Reference Library of Science and Invention. This is certainly a project needing imaginative and sympathetic consideration, not only by librarians but also even more, perhaps, by the scientific societies and institutions and by technical interests in industry. It is earnestly to be hoped that, as representatives of potential users of the new library, they will study the plans and give their considered views soon, lest the preparatory work becomes too far advanced for them to be taken into account.

The success of the undertaking will, however, depend primarily on the degree to which the Government itself demonstrates its determination to treat the communication of research results, and technical knowledge generally, with the same seriousness and sense of urgency as are devoted to the acquisition of them. In this, the new Library could prove a most potent instrument. But on its two fundamental needs-adequate space to house the necessary documents and adequate staff to exploit them-Mr. Freeth's reply to the adjournment debate in the House of Commons on March 14 was anything but reassuring. The decision to split the holdings of the new Library between the British Museum site in Bloomsbury and the new building on the South Bank, while regrettable, is understandable. But it can only be regarded as a workable compromise on 